\title{
高分子化学缩聚反应教学中若干问题的探讨
}

\author{
孟志芬* \\ 河南科技学院化学化工学院, 河南 新乡 453003
}

摘要: 高分子化学缩聚反应教学中有几个比较容易困扰学生的问题, 如基团数比 $(r)$ 值的含义、聚酯化线形平衡缩聚 动力学方程的推导与应用等, 本文结合多年的教学实践经验, 对这些问题进行了探讨和梳理, 可为同行教学和学生 学习提供一定的参考。

关键词: 缩聚反应; 基团数比 $r$; 聚酯化线形平衡缩聚动力学方程

中图分类号: G64; O6

\section{Exploration on Some Problems about Condensation Polymerization in Polymer Chemistry Teaching}

\author{
Zhifen Meng * \\ Department of Chemistry and Chemical Engineering, Henan Institute of Science and Technology, Xinxiang 453003, \\ Henan Province, China.
}

\begin{abstract}
There are some problems in the condensation polymerization teaching, such as the ratio of function groups, the Polyester linear equilibrium polycondensation kinetic equation, etc. The students were often puzzled with these problems. Based on many years of polymer chemistry teaching experience, these problems were discussed in this paper to provide some reference for peers teaching and students learning.
\end{abstract}

Key Words: Condensation polymerization; The ratio of function groups;

The polyester linear equilibrium polycondensation kinetic equation

高分子化学是高分子材料和相关专业学生必修的专业基础课, 国内外有不少以 “高分子化学” 命名的教材或专著, 目前国内高校大多采用潘祖仁主编的《高分子化学》(以下简称潘版教材)为教 材。高分子化学理论性强、概念抽象, 学生学习较为吃力。另外高分子化学发展历史较短, 人们对 很多领域的认识和理论研究尚不完善, 存在争议, 也有一些概念表达不是特别清楚和全面, 于是有 多种不同版本的教材和学习指导书对一些内容进行解读和分析, 但也出现了一些错误与不足, 给学 生自主学习造成了一定的困惑和烦恼, 因此教师在教学过程中如何讲解这些容易让学生产生困扰的 知识点就显得特别重要。笔者根据多年的教学实践, 对缩聚反应教学中基团数比、聚酯化可逆平衡 线性缩聚动力学推导等问题谈谈自己的认识和看法。

\section{1 关于 $r$ 值的含义}

潘版教材在讲基团数比对聚合度的影响时提到 “实际上, 总是在两基团数不相等的条件下操作,

收稿: 2020-01-02; 录用: 2020-03-24; 网络发表: 2020-04-09

“通讯作者, Email: mengzf@yeah.net

基金资助：河南省高等学校合格基层教学组织建设项目 
进行理论分析时, 需引入两种单体的基团数比或摩尔比 $r$ ”, 据此很多学生认为 $r$ 既可理解为两种单 体的基团数比也可理解为两种单体的摩尔比。但在潘版教材中, 又以二元酸 $(\mathrm{aAa})$ 和二元醇 $(\mathrm{bBb})$ 进行 缩聚为例进一步说明 $r$ 值的含义。设 $N_{\mathrm{a}} 、 N_{\mathrm{b}}$ 为 $\mathrm{a} 、 \mathrm{~b}$ 的起始基团数, 分别为两种单体分子数的 2 倍, 按定义, 设 $r=N_{\mathrm{a}} / N_{\mathrm{b}} \leq 1$, 并且在接下来的对两基团数不相等和相等的两种情况加以分析时明确指出 $r$ 为基团数比。比如, 有 “据此, 就可以设定基团数比 $r$ 来控制聚合度” “包括 2-2 体系或 2 体系, 另加微量单官能团物质 $\mathrm{Cb}$, 则按下式计算基团数比 $r$ ” 等描述 ${ }^{[1]}$ 。由此可见, 在潘版教材中, $r$ 为基 团数比。

王槐三等编著《高分子化学教程》(以下简称王版教材)相对分子质量控制方法中有以下描述。设 缩聚反应: 反应开始时两种单体的物质的量分别为 $N_{\mathrm{a}} 、 N_{\mathrm{b}}$, 且 $N_{\mathrm{a}} \leq N_{\mathrm{b}}$, 则两种官能团的物质的量分 别为 $2 N_{\mathrm{a}}$ 和 $2 N_{\mathrm{b}}$, 同时设 $N_{\mathrm{a}} / N_{\mathrm{b}}=r \leq 1$ 。这样的描述给人的感觉是 $r$ 为两种单体的物质的量之比, 并 且在接下来的推导中将 $r=N_{\mathrm{a}} / N_{\mathrm{b}}$ 代入, 得出线形缩聚平衡反应中聚合度与起始单体物质的量配比及 反应程度之间的重要关系式, 即公式(1)。

$$
\overline{X_{n}}=\frac{1+r}{1+r-2 r p}
$$

但在涉及公式(1)的有关要点讨论时, 又将 $r$ 称作 “官能团摩尔系数” , 并进一步说明它是数值 小的官能团物质的量与数值大的官能团物质的量之比, 它一定小于或等于 1 , 绝无出现大于 1 的情 况 ${ }^{[2]}$ 。由此可见, 王版教材 $r$ 值实际表达的意义也是官能团数之比。两教材中 $r$ 值的表达式同样都是 $r=N_{\mathrm{a}} / N_{\mathrm{b}}$, 但潘版教材中代表了两种官能团的物质的量之比, 而王版教材却是两种单体的物质的量 之比。

对于由单体 $\mathrm{aAa}$ 和 $\mathrm{bBb}$ 组成的 2-2 官能度体系, 官能团数之比与单体的物质的量之比相等, 这 两种定义的计算结果并无差别。如:

例 1: $1 \mathrm{~mol}$ 对苯二甲酸与 $1.02 \mathrm{~mol}$ 乙二醇反应得到聚酯, 求反应程度 $p$ 为 0.998 时的数均聚合 度。

若 $r$ 为基团数比, 则: $r=\frac{N_{\mathrm{a}}}{N_{\mathrm{b}}}=\frac{1 \times 2}{1.02 \times 2}=0.98$

若 $r$ 为单体物质的量之比, 则: $r=\frac{N_{\mathrm{a}}}{N_{\mathrm{b}}}=\frac{1}{1.02}=0.98$

在例 1 中, $r$ 值两种定义的计算结果是一样的, 当然根据公式(1)求出的数均聚合度也是相同的。 教材上的不同表述和类似例 1 题目的计算结果, 导致一些学生认为 $r$ 既可理解为两种单体的基团数 比也可理解为两种单体的物质的量之比，二者计算结果相同。

但对于由 $\mathrm{aAa}$ 和 $\mathrm{bBb}$ 或 $\mathrm{aRb}$ 、另加微量单官能团物质 $\mathrm{Cb}$ 组成的体系, $r$ 值两种定义的计算结果 是不相同的, 把 $r$ 值按两种单体物质的量之比计算, 求出的数均聚合度数值是错误的。如:

例 2: 等摩尔二元醇和二元酸缩聚, 另加醋酸 $1.5 \%$, 求 $p=0.995$ 时的聚合度是多少?

$$
r=\frac{N_{\mathrm{a}}}{N_{\mathrm{b}}+2 N_{\mathrm{b}}^{\prime}}
$$

公式(2)中, $N_{\mathrm{b}}^{\prime}$ 为单官能团物质 $\mathrm{Cb}$ 的基团数。根据公式(2)和(1)分别计算 $r$ 值和数均聚合度如 下:

若 $r$ 为基团数比, 计算得: $r=0.985 ; \overline{X_{n}}=79.4$ 。

若 $r$ 为单体物质的量之比, 计算得: $r=0.971 ; \overline{X_{n}}=50.5$ 。

由例 2 可以看出, 对 $r$ 值的两种不同理解, 产生了两种不同的计算结果。那么到底该如何定义 $r$ 值呢? 根据潘版教材对公式(1)的推导过程可知, $r$ 值表示的是两种双官能团单体的官能团数之比, 并且在线形缩聚平衡反应中, 多数教材表示聚合度与起始单体物质的量配比及反应程度之间的关系 
均采用公式(1)。因此, 应将 $r$ 值定义为两种单体的基团数之比, 即 $N_{\mathrm{a}} 、 N_{\mathrm{b}}$ 分别为官能团 $\mathrm{a} 、 \mathrm{~b}$ 的起 始基团数, 而不是两种单体的物质的量。

针对目前教材对 $r$ 值的不同表述, 笔者建议, 教师在授课过程中, 一是需要给学生强调 $r$ 为基 团数之比而不是单体分子数之比; 二是通过举例让学生明白 $r$ 值两种表述的差异; 三是可以将 $r$ 值 的两种不同定义作为问题提出, 引导学生在推导公式(1)的过程中得出结论。另外, 笔者也对教材提 一点拙见, 建议编者对理解一致、没有争议的重要概念最好能够统一、严谨地表达, 避免给学生自 主学习造成干扰与困惑。

\section{2 关于基团数比 $r=N_{a} /\left(N_{b}+2 N_{b}^{\prime}\right)$ 中 “ 2 ” 的意义}

对于由 $\mathrm{aAa}$ 和 $\mathrm{bBb}$ 或 $\mathrm{aRb}$, 另加微量单官能团物质 $\mathrm{Cb}$ 组成的体系, $r$ 值按公式(2)计算。公 式(2)分母中的 “ 2 ”, 潘版教材是这样解释的: “2” 表示 1 分子单基团 b 的 $\mathrm{Cb}$ 与双官能团的过量 $\mathrm{bBb}$ 相当, 因为过量的 $\mathrm{bBb}$ 只有一个基团 $\mathrm{b}$ 起封端作用, 另一基团 $\mathrm{b}$ 不起作用, 类似单官能团 $\mathrm{Cb}{ }^{[1]}$ 。很多学生不理解这种说法, 认为既然 1 分子单基团 $\mathrm{b}$ 的 $\mathrm{Cb}$ 与双官能团的过量 $\mathrm{bBb}$ 相当, 就 不必加 2 , 直接是 1 就可以了。正因为不能准确理解公式(2)分母中 “2” 的含义, 导致学生在做相关 题目时容易漏掉 “2”而出现计算错误。

其实, 教材表达的意思是一个 $\mathrm{Cb}$ 分子与一个过量的 $\mathrm{bBb}$ 在限制聚合物链增长方面具有同样的 定量影响。潘版教材在 $\mathrm{aAa}$ 和 $\mathrm{bBb}$ 体系公式推导过程中, 利用了聚合物链端的总数等于未反应 $\mathrm{a} 、$ $\mathrm{b}$ 官能团数的总和。因为每个聚合物链有两个链端, 所以聚合物分子总数等于未反应 $\mathrm{a} 、 \mathrm{~b}$ 官能团总 数的一半。一个过量的 $\mathrm{bBb}$ 封端, 链端还有一个未反应的基团 $\mathrm{b}$, 而一个 $\mathrm{Cb}$ 封端后链端一般是烃 基, 不再有官能团, 也就是说 $\mathrm{Cb}$ 封端产生的大分子链端只有一个未反应的官能团。而实际推导过 程中是按每个大分子链两端有两个未反应的官能团处理, 聚合物分子总数等于未反应 $\mathrm{a} 、 \mathrm{~b}$ 官能团总 数的一半, 所以 $N_{\mathrm{b}}^{\prime}$ 前必须加 2 。可是潘版教材上只有 $\mathrm{aAa}$ 和 $\mathrm{bBb}$ 体系公式推导过程, 没有 $\mathrm{aAa}$ 和 $\mathrm{bBb}$ 或 $\mathrm{aRb}$ 、另加微量单官能团物质 $\mathrm{Cb}$ 组成体系的公式推导过程。由于高聚物聚合机理和过程比较 抽象, 教师在课堂上仅用上述语言进行解释, 教学效果并不是太好。笔者建议, 在讲这部分内容时, 增加 $\mathrm{aAa}$ 和 $\mathrm{bBb}$ 、另加微量单官能团物质 $\mathrm{Cb}$ 组成体系的数均聚合度公式推导过程, 这样学生能够 清楚地看到当推导出相同的公式(1)时, $r$ 值的表达形式是公式(2), 这时再加上语言解释, 更有利于 学生理解和掌握公式(2)。该体系推导过程可参考文献[3]。另外, 还可以将 $\mathrm{aRb}$ 、另加微量单官能团 物质 $\mathrm{Cb}$ 组成体系的数均聚合度公式推导过程作为课后作业留给学生自己去推导, 使学生能够举一 反三、融会贯通, 并深刻理解公式的推导过程和相关参数所代表的含义。对于教材, 笔者建议编者 增加 $\mathrm{aAa}$ 和 $\mathrm{bBb}$ 、另加微量单官能团物质 $\mathrm{Cb}$ 组成体系的数均聚合度公式的推导过程。

\section{3 关于聚酯化线形平衡缩聚动力学方程的推导与应用}

潘版教材在讨论反应程度和平衡常数对聚合度的影响时, 推导出了线形平衡缩聚反应方程, 如 公式(3)所示。

$$
\overline{X_{n}} \approx \sqrt{\frac{K}{n_{\mathrm{w}}}}
$$

公式(3)建立起了在开放体系中进行的线形平衡缩聚反应产物的数均聚合度与平衡常数以及体 系中小分子含量之间的近似定量关系。关于平衡缩聚反应体系中小分子含量的计算问题主要有两种, 一是求取小分子的含量, 二是求取小分子的残留率。如:

例 3: 等摩尔的乙二醇和对苯二甲酸在 $280^{\circ} \mathrm{C}$ 下封管内进行缩聚, 平衡常数 $K=4$, 欲使产物数 均聚合度为 100 , 问体系中残留水分有多少?

例 4: 等摩尔的乙二醇和对苯二甲酸 $280^{\circ} \mathrm{C}$ 下封管内进行缩聚, 平衡常数 $K=6.6$, 如达平衡时 
所得产物的数均聚合度为 120 , 问体系中残存的小分子所占的分数有多少?

显然例 3 是问体系中残留水分的量是多少，单位应是 $\mathrm{mol}$ 或 $\mathrm{mol} \cdot \mathrm{L}^{-1}$; 例 4 是问体系中小分子的 残留率, 是个比值, 没有单位。含义迥异的两个问题却都用公式(3)去求解, 学生对此往往深感疑惑, 公式中的 $n_{\mathrm{w}}$ 到底是指什么?

潘版教材在推导线形缩聚动力学方程时, 假定羟基或羧基的起始浓度 $c_{0}=1$, 这种处理简化了 推导过程, 但所得出的线形缩聚平衡方程却不能反映羟基或羧基起始浓度对产物数均聚合度以及体 系中小分子残留量的影响。学生在用该方程解答没有给出单体起始浓度的等物质的量的聚合体系的 相关问题时, 一般会仿照教材按羟基或羧基的起始浓度 $c_{0}=1$ 处理。但是对于一些 $c_{0}$ 不为 1 的体系, 学生因为不清楚单体浓度对聚合度和小分子残留量有何影响, 往往不知道如何计算。如:

例 5: $2 \mathrm{~mol}$ 己二酸和 $2 \mathrm{~mol}$ 已二胺进行缩聚, 已知聚合-解聚平衡常数为 400, 试问不脱水时聚 合度最高可以达到多少? 如欲使聚合度达到 1000 , 则体系中的水应该脱除到什么程度?

总之, 该线形缩聚平衡方程在具体应用上给学生造成的困惑主要有两点: 一是 $n_{\mathrm{w}}$ 的含义; 二是 单体起始浓度对数均聚合度的影响以及官能团起始浓度和小分子残留量之间的关系。

关于 $n_{\mathrm{w}}$, 不同版本的教材和学习指导书大致有两种表述, 一种是潘版教材在推导线形缩聚动力 学方程时, 设残留水的浓度为 $n_{\mathrm{w}}$, 即 $n_{\mathrm{w}}$ 为体系中残留水的浓度。贾红兵主编《高分子化学导读与题 解》中也有类似表达, “ $n_{\mathrm{w}}$ 为生成小分子副产物的残留量” [4]。另一种是王版教材中的定义, $n_{\mathrm{w}}=$ $N_{\mathrm{w}} / N_{0}$, 将 $n_{\mathrm{w}}$ 定义为存留在体系中小分子的摩尔分数, 或者叫做 “小分子存留率”。它的含义是指 实际存留在聚合反应体系中的小分子物质的量与理论上能够生成的小分子物质的量的比值, $N_{\mathrm{w}}$ 为实 际存留在聚合反应体系中小分子的物质的量, $N_{0}$ 为理论上能够生成小分子的物质的量, 即起始羟基 或羧基的物质的量, 也就是潘版教材中的 $c_{0}$ 。何旭敏等 ${ }^{[5]}$ 编《高分子化学学习指导》也是这种表述。 从两种定义可以看出, 前者为体系中残留水的浓度, 更确切的说应该是体系中残留水的物质的量, 单位一般为 $\mathrm{mol}$, 后者是体系中小分子存留率, 是个无量纲的比值。二者孰对孰错? 上述题目到底 该如何解决?

笔者认为不妨将动力学方程推导过程还原为最普通的情况, 假定 $N_{\mathrm{w}}$ 为实际存留在聚合反应体 系中小分子的物质的量, 以羟基或羒基起始的物质的量为 $c_{0}$ 进行推导, 这样得出的公式具有普适性, 不仅能够搞清楚 $n_{\mathrm{w}}$ 的含义, 还能明确单体起始的物质的量对产物聚合度和小分子含量的影响, 这样 学生无论遇到什么样的题目都能顺利解答。具体推导过程如下:

$$
-\mathrm{COOH}+\mathrm{HO}-\underset{k_{-1}}{\stackrel{k_{1}}{\rightleftharpoons}}-\mathrm{OCO}-+\mathrm{H}_{2} \mathrm{O}
$$

起始

$t$ 时, 水未排除

$t$ 时，水部分排除

$c_{0} \quad c_{0}$

$\begin{array}{llll}c & c & c_{0}-c & c_{0}-c \\ c & c & c_{0}-c & N_{\mathrm{w}}\end{array}$

聚酯反应的总速率是正、逆反应速率之差。水未排除时, 速率为:

$$
R=-\frac{\mathrm{d} c}{\mathrm{~d} t}=k_{1} c^{2}-k_{-1}\left(c_{0}-c\right)^{2}
$$

水部分排除时的总速率为:

$$
\begin{aligned}
& R=-\frac{\mathrm{d} c}{\mathrm{~d} t}=k_{1} c^{2}-k_{-1}\left(c_{0}-c\right) N_{w} \\
& \because p=\frac{N_{0}-N}{N_{0}}=1-\frac{N}{N_{0}}=1-\frac{c}{c_{0}} ; \therefore c=c_{0}(1-p)
\end{aligned}
$$

将式(6)和平衡常数 $K=k_{1} / k_{-1}$ 代入式(4), 得:

$$
-\frac{\mathrm{d} c}{\mathrm{~d} t}=k_{1} c_{0}^{2}(1-p)^{2}-\frac{k_{1}}{K} c_{0}^{2} p^{2}
$$




$$
-\frac{\mathrm{d} c}{\mathrm{~d} t}=k_{1} c_{0}^{2}\left[(1-p)^{2}-\frac{p^{2}}{K}\right]
$$

将式(6)和平衡常数 $K=k_{1} / k_{-1}$ 代入式(5), 得:

$$
\begin{aligned}
& -\frac{\mathrm{d} c}{\mathrm{~d} t}=k_{1} c_{0}^{2}(1-p)^{2}-\frac{k_{1}}{K} c_{0} p N_{\mathrm{w}} \\
& -\frac{\mathrm{d} c}{\mathrm{~d} t}=k_{1} c_{0}^{2}\left[(1-p)^{2}-\frac{p N_{\mathrm{w}}}{K c_{0}}\right]
\end{aligned}
$$

聚酯化反应是可逆反应, 反应平衡时, 总速率等于零, 对于密闭体系, 由式(8)得:

$$
\begin{aligned}
& k_{1} c_{0}^{2}\left[(1-p)^{2}-\frac{p^{2}}{K}\right]=0 \\
& (1-p)^{2}-\frac{p^{2}}{K}=0
\end{aligned}
$$

解得:

$$
\begin{aligned}
& p=\frac{\sqrt{K}}{\sqrt{K}+1} \\
& \overline{X_{n}}=\sqrt{K}+1
\end{aligned}
$$

由式(13)、(14)可以看出, 对于密闭体系, 线形平衡缩聚反应达到平衡时的反应程度和产物的数 均聚合度仅由平衡常数决定, 与单体的起始浓度无关。

对于敞开体系, 由式(10)得：

$$
\begin{aligned}
& k_{1} c_{0}^{2}\left[(1-p)^{2}-\frac{p N_{\mathrm{w}}}{K c_{0}}\right]=0 \\
& (1-p)^{2}-\frac{p N_{\mathrm{w}}}{K c_{0}}=0 \\
& \overline{X_{n}}=\frac{1}{1-p}
\end{aligned}
$$

将式(17)代入式(16)得:

$$
\overline{X_{n}}=\sqrt{\frac{K c_{0}}{p N_{\mathrm{w}}}}
$$

令 $n_{\mathrm{w}}=N_{\mathrm{w}} / c_{0}$, 则式(18) 可写为:

$$
\overline{X_{n}}=\sqrt{\frac{K}{p n_{\mathrm{w}}}} \approx \sqrt{\frac{K}{n_{\mathrm{w}}}}
$$

从表达形式上来看, 上述推导结果式(19)和多数教材及指导书是一致的。从上述推导过程可以 看出, 在式(19)中, $n_{\mathrm{w}}$ 是体系中小分子的存留率, 是个无量纲的比值, 而不是体系中小分子的物质 的量。如果需要求出体系中小分子的浓度 $N_{\mathrm{w}}$, 根据 $n_{\mathrm{w}}=N_{\mathrm{w}} / c_{0}$, 即可求出。因此, 对于没有给出单 体起始物质的量的体系, 根据线形缩聚平衡方程, 只能求出体系中小分子的存留率, 而不能求出体 系中小分子的物质的量。比如, 例 3 和例 4 虽然问法不同, 但二者都是没有给出单体起始物质的量 的体系, 即 $c_{0}$ 不确定, 因此都只能求出体系中小分子的存留率, 而不能求出体系中小分子的物质的 量。在例 5 中, 两种单体已二酸和己二胺起始物质的量都是 $2 \mathrm{~mol}$, 即羧基或氨基起始物质的量 $c_{0}$ 为 $4 \mathrm{~mol}$, 所以该题目可以根据线形缩聚平衡方程, 求出体系中小分子的存留率 $n_{\mathrm{w}}$, 还可以进一步 根据 $n_{\mathrm{w}}=N_{\mathrm{w}} / c_{0}$, 求出体系中小分子的浓度 $N_{\mathrm{w}}$ 。

在潘版教材线形缩聚平衡方程推导过程中, 因为假定了羟基或羧基的起始浓度 $c_{0}=1$, 所以 
$n_{\mathrm{w}}=N_{\mathrm{w}} / c_{0}=N_{\mathrm{w}}$, 从形式和数值上看二者相等, 但实际代表的意义并不相同。

基于以上讨论, 对于这部分内容, 笔者认为, 作为教师可以采用两种方法进行处理, 一是按照 上述方法推导线形缩聚平衡方程, 从根本上解决学生对 $n_{\mathrm{w}}$ 的含义以及单体起始浓度与小分子残留量 之间关系的困惑; 同时也可以让学生把两种推导方法进行对比, 能够加深学生对该部分内容的理解 与把握。二是在学习教材推导方法的基础上, 通过给出一些题目让学生解答, 鼓励学生大胆质疑, 启发学生发现问题, 并进一步引导学生按照上述方法推导线形缩聚平衡方程解决问题, 从而培养学 生自主学习的能力。

对于 $n_{\mathrm{w}}$, 目前不仅存在不同版本的教材或学习指导书对其含义表述的不一致性, 并且也有教材 用 $P_{\mathrm{w}}$ 来表示体系中小分子的含量, 其线形缩聚平衡方程表达式和式(19)不相同。例如, 在唐黎明 等 ${ }^{[6]}$ 编著的《高分子化学》中, 线形缩聚平衡方程为式(20)。对于各种教材在某些概念或公式上的不 一致性, 笔者建议各位主编以权威教材为蓝本, 在科学性和合理性的基础上, 尽量保持重要概念和 公式的一致性; 另外, 如果各位同仁认可以上讨论, 笔者也建议教材再版时, 可以考虑假定羟基或 羧基的起始浓度为 $c_{0}$ 推导线形缩聚平衡方程。

$$
\overline{X_{n}}=\sqrt{\frac{K}{P P_{\mathrm{w}}}}
$$

\section{4 结语}

总之, 由于人们对高分子化学很多领域的理论研究尚不完善, 在概念表述及公式推导上存在不 足与争议是新学科发展中的正常现象。如果教师在课堂教学中能够不拘泥于教材, 引导学生将有待 完善的知识点以问题的形式提出, 并指导学生以探究式的学习方法尝试解决这些问题, 不仅有助于 完善高分子化学某些具体的知识和理论, 更有利于培养学生的创新意识和创新能力。

\section{参 考 文 献}

[1] 潘祖仁. 高分子化学. 第 5 版. 北京: 化学工业出版社, 2011: 28-34, 19-20, 34-35.

[2] 王槐三, 寇晓康. 高分子化学教程. 北京: 科学出版社, 2002: 53-58.

[3] 杨建国. 高分子通报, 2011, No. 1, 109.

[4] 贾红兵. 高分子化学导读与题解. 北京: 化学工业出版社, 2010: 25 .

[5] 何旭敏, 董炎明. 高分子化学学习指导. 北京: 科学出版社, 2007: 157-158.

[6] 唐黎明, 度新林. 高分子化学. 清华大学出版社, 2009: 267-270. 